\title{
Viscosity Destabilizes Sonoluminescing Bubbles
}

\author{
Ruediger Toegel, Stefan Luther, and Detlef Lohse \\ Faculty of Science \& Technology, University of Twente, Post Office Box 217, 7500 AE Enschede, The Netherlands
}

(Received 27 July 2005; published 20 March 2006)

\begin{abstract}
In single-bubble sonoluminescence (SBSL) microbubbles are trapped in a standing sound wave, typically in water or water-glycerol mixtures. However, in viscous liquids such as glycol, methylformamide, or sulphuric acid it is not possible to trap the bubble in a stable position. This is very peculiar as larger viscosity normally stabilizes the dynamics. Suslick and co-workers call this new mysterious state of SBSL "moving-SBSL." We identify the history force (a force nonlocal in time) as the origin of this destabilization and show that the instability is parametric. A force balance model quantitatively accounts for the observed quasiperiodic bubble trajectories.
\end{abstract}

DOI: 10.1103/PhysRevLett.96.114301

In 1989 Gaitan succeeded in capturing a single light emitting bubble in a standing sound wave in a waterglycerol mixture. The phenomenon is called single-bubble sonoluminescence (SBSL) [1-12]. In their recent work Suslick and co-workers report that in liquids with large viscosity a new mysterious state of single-bubble sonoluminescence (SBSL) can be observed [3]: In contrast to less viscous liquids the bubbles are not spatially stationary anymore but show quasiperiodic, often circular translational motion on a time scale of seconds. Accordingly, Suslick and co-workers call the phenomenon movingSBSL (m-SBSL). The aim of the present work is to theoretically account for this new state of SBSL. This Letter is organized as follows: We first briefly describe our measurements and the processing of the experimental data; we then develop a force balance model for the bubble's translational motion with effective forces [13], namely, drag, buoyancy, added mass, and, in particular, the history force as described in Ref. [14]. The experimental data and the predictions of our model are finally combined in a phase diagram which compares theory and experiment to each other showing excellent agreement.

Experiment. - We inject a single bubble of typical ambient radius $R_{0}=40 \mu \mathrm{m}$ into a standard sonoluminescence flask (radius of the flask $R_{f l}=3 \mathrm{~cm}$ ) filled with degassed glycol $\left(\nu=20 \times 10^{-6} \mathrm{~m}^{2} / \mathrm{s}\right)$. The flask is driven at its lowest resonance frequency of $f \simeq 23 \mathrm{kHz}$. In contrast to the water case $\left(\nu=10^{-6} \mathrm{~m}^{2} / \mathrm{s}\right)$ or other cases with lower viscosity where it is easy to trap the gas bubble in the center, here the bubble cannot be trapped but moves on an (often slowly drifting) ellipsoidal trajectory with typical diameter of $1 \mathrm{~mm}$. An example is shown in Fig. 1, lower right panels. It should be noted that due to the high viscosity of glycol the above described path instabilities set in at moderate acoustical forcing already $\left(P_{a} \simeq 0.8\right.$ bar $)$ - unlike in the experiments of Ref. [3]. Accordingly, we do not observe any light emission. We obtain the kinematics of the motion by simultaneous tracking of the bubble's translational motion with a high speed camera (2000 frames per second) and its radial oscillations by means of Mie-scattering technique. The Mie signal
PACS numbers: $78.60 . \mathrm{Mq}$

subsequently is fitted to a Rayleigh-Plesset-type equation of motion [cf. Equation (4)] with two fit parameters - the ambient radius $R_{0}$ of the moving bubble and the driving pressure $P_{a}$ of the sound field.

Modeling the acoustics. - The external pressure field the bubble is subjected to is rather complex. It depends on acoustical and geometrical properties of the flask like its acoustical impedance or its symmetry. Assuming for the time being spherical symmetry and a node at the flasks surface, the pressure field of the lowest resonance is given by the spherical Bessel function $j_{0}$

$$
p_{a c}(\mathbf{x}, t)=P_{a} \sin \omega t j_{0}\left(\frac{\pi|\mathbf{x}|}{R_{f l}}\right),
$$

with $P_{a}$ the center pressure, $\omega=2 \pi f$ the (angular) resonance frequency of the field, $\mathbf{x}$ the position of the bubble (with respect to the center), and $R_{f l}$ the radius of the flask. Since in addition we are only interested in small distances $|\mathbf{x}|$, Eq. (1) can be Taylor-expanded, giving

$$
p_{a c}(\mathbf{x}, t)=-P_{a} \sin (\omega t)\left(1-\frac{\pi^{2}|\mathbf{x}|^{2}}{6 R_{f l}^{2}}\right) .
$$

As mentioned above the acoustical field in experiment is not perfectly spherically symmmetric but somewhat distorted as is confirmed by measurements of the spatial pressure distribution. Though these distortions have no influence on the overall features described in the present work they do cause symmetry breaking; i.e., they are responsible for the 3D structure of a bubble's trajectory. Where necessary, we conveniently account for such distortions by mapping the original spherically symmetric field onto an ellipsoidal one with (dimensionless) main axis $a_{1}, a_{2}, a_{3}: \quad|\mathbf{x}|=\sqrt{\left(x_{1} / a_{1}\right)^{2}+\left(x_{2} / a_{2}\right)^{2}+\left(x_{3} / a_{3}\right)^{2}}$. The velocity field of the standing wave is readily obtained from Eq. (2). We compute it from the linearized, inviscid momentum balance, $\rho \partial_{t} \mathbf{u}=-\nabla p_{a c}$, yielding

$$
u_{i}(\mathbf{x}, t)=\frac{\pi^{2} x_{i} P_{a}}{3 \rho \omega a_{i}^{2} R_{f l}^{2}} \cos \omega t
$$

The radial motion of the bubble is described by a Rayleigh- 

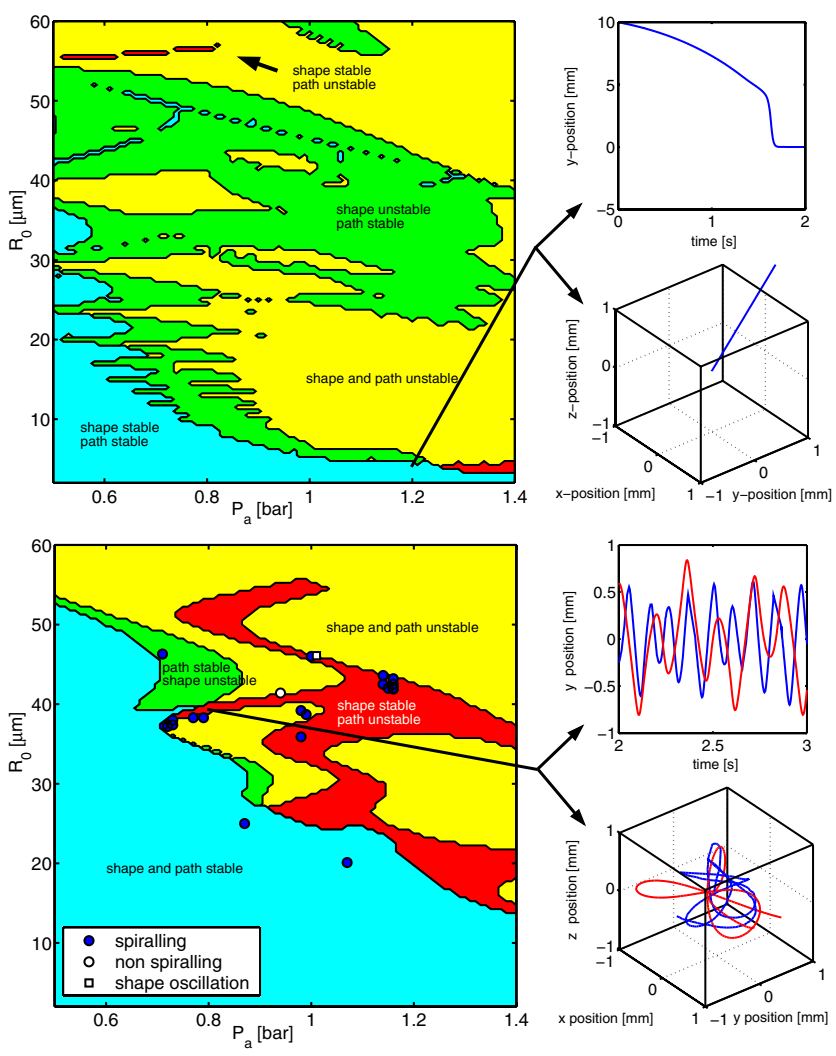

FIG. 1 (color online). Phase diagrams in the ambient radius $R_{0}$ vs driving pressure $P_{a}$ phase space for the bubbles in water $(\nu=$ $10^{-6} \mathrm{~m}^{2} / \mathrm{s}$, upper) and glycol $\left(\nu=20 \times 10^{-6} \mathrm{~m}^{2} / \mathrm{s}\right.$, lower) for a driving frequency of $f=23 \mathrm{kHz}$ and a liquid temperature of $T=18^{\circ} \mathrm{C}$. In the lower left area (blue online) the bubbles are shape and path stable. In the medium gray area (green online) the bubbles are shape unstable and path stable. In the light gray area (yellow online) they are both shape and path unstable. Hence, in the medium and light gray area bubbles cannot exist. In the glycol case there is a new and additional phase (dark gray, red online) of shape stable, but path unstable bubble which we identify with the observed m-SBSL. Here the bubbles spiral out of the center of the flask, as demonstrated through the threedimensional bubble trace in the physical $(x, y, z)$ space (lower right) and its projection $y(t)$ : dark (blue online) curves correspond to our experimental observations, lighter (red online) curves to the result of our model. The corresponding curves for the water case show very different behavior, namely, only a bubble moving to the center of the cell (upper right). In the phase diagram for glycol additional symbols are plotted. They mark our experimental data points. Dark (blue online) circles denote moving bubbles, the single white square a bubble where additional shape oscillation could be observed and the single white circle a still bubble. The agreement with the predictions from the theoretical phase diagram is very good; i.e., the blue circles are all inside or close to the dark (red online) area. Note that we have found various nonspiralling bubbles (white circles) in the blue area (where they belong), but as simultaneously shape- and pathstable bubbles are well understood, for clarity we did not include these points in the diagrams. Only one nonspiralling shape stable bubble is slightly off the blue regime (white circle at 0.95 bar and $40 \mu \mathrm{m}$ in the lower figure). The reason may be that the Mathieu tongue around $40 \mu \mathrm{m}$ is less pronounced in our model as compared to the experiments.
Plesset type equation [15], which takes into account first order corrections for the liquid compressibility [16]:

$$
\begin{aligned}
\left(1-\frac{\dot{R}}{c}\right) R \ddot{R}+\frac{3}{2} \dot{R}^{2}\left(1-\frac{\dot{R}}{3 c}\right)= & \left(1+\frac{\dot{R}}{c}\right) \frac{1}{\rho}\left(p_{g}-p_{a c}-P_{0}\right) \\
& +\frac{R \dot{p}_{g}}{\rho c}-\frac{4 \nu \dot{R}}{R}-\frac{2 \gamma}{\rho R}
\end{aligned}
$$

Here, $\rho, c, \gamma$, and $\nu$ are the density, speed of sound, surface tension, and viscosity of the liquid. We investigate two different liquids - water and ethylene glycol. A collection of the corresponding material constants is shown in Table I. In addition, $p_{g}$ denotes the gas pressure at the bubble surface, $P_{0}=1.013$ bar the ambient pressure, and $p_{a c}(\mathbf{x}, t)$ the local pressure of the acoustic driving according to Eqs. (2). Note that Eq. (4) is derived under the assumption of spatial homogeneity. In our case this is, strictly speaking, not the case because $p_{a c}=p_{a c}(\mathbf{x}, t)$. However, spatial changes of $p_{a c}$ occur on a length scale of the order of $R_{f l}$. The typical change of position of the bubble during one cycle turns out to be much smaller than that (submicrons) such that the assumption of spatial homogeneity a posteriori is fairly well fulfilled.

Equation (4) needs to be closed with a suitable expression for the gas pressure at the bubble surface. In accordance with Ref. [18], we use an isothermal, van der Waalstype equation of state,

$$
p_{g}=\frac{N k T_{0}}{V-N B}+p_{\text {vap }},
$$

with $N$ the number of particles of the bubble, $V$ its (time dependent) volume, $T_{0}=291 \mathrm{~K}$ the ambient temperature, $N B$ the excluded volume of the gas, and $p_{\text {vap }}$ the equilibrium vapor pressure of the liquid.

Force balance model. - Asymptotic expressions for the hydrodynamic force on a bubble which is exposed to an external velocity field and the radius of which in addition varies with time analytically have been obtained in Ref. [14]. Based on the results of that reference we now derive an equation for the translational motion of the bubble which combines those asymptotic limits. Our method also offers a way to efficiently handle the history integral, in order to avoid the numerical difficulties associated with the temporal nonlocality of the ordinary differential equation (ODE).

Given the relative velocity between the fluid and the bubble, $\mathbf{U}=\mathbf{u}-\mathbf{v}$, and the bubble wall speed $\dot{R}$, two limiting cases are distinguished in Ref. [14].

TABLE I. material constants of ethylene glycol and water taken from Ref. [17]. The vapor pressure of ethylene glycol is negligible. Hence we use $p_{\text {vap }}=0 \mathrm{~Pa}$.

\begin{tabular}{lccccc}
\hline \hline \multicolumn{1}{c}{ liquid } & $c\left[\frac{\mathrm{m}}{\mathrm{s}}\right]$ & $\rho\left[\frac{\mathrm{kg}}{\mathrm{m}^{3}}\right]$ & $\nu\left[\frac{\mathrm{m}^{2}}{\mathrm{~s}}\right]$ & $\gamma\left[\frac{\mathrm{N}}{\mathrm{m}}\right]$ & $p_{\text {vap }}[\mathrm{Pa}]$ \\
ethylene glycol & 1660 & 1100 & $19 \times 10^{-6}$ & $45 \times 10^{-3}$ & 0 \\
water & 1485 & 1000 & $1 \times 10^{-6}$ & $72 \times 10^{-3}$ & $2.3 \times 10^{3}$ \\
\hline \hline
\end{tabular}


(i) Both Reynolds numbers, the translationally based one, $R e_{t}=R|\mathbf{U}| / \nu$, as well as the radially based one, $R e_{r}=$ $R|\dot{R}| / \nu$, are small. In this case the total force acting on the bubble is found to be

$$
\begin{aligned}
\mathbf{F}(t)= & 4 \pi \rho \nu R(t) \mathbf{U}(t)+\frac{4}{3} \pi \rho R(t)^{3} \frac{d \mathbf{v}}{d t}-\frac{4}{3} \pi \rho R(t)^{3} \mathbf{g}+\frac{2}{3} \pi \rho\left(\frac{d\left[R(t)^{3} \mathbf{U}(t)\right]}{d t}+2 R(t)^{3} \frac{d \mathbf{U}(t)}{d t}\right) \\
& +8 \pi \rho \nu \int_{0}^{t} \exp \left[9 \nu \int_{\tau}^{t} R\left(t^{\prime}\right)^{-2} d t^{\prime}\right] \operatorname{erfc}\left[\sqrt{9 \nu \int_{\tau}^{t} R\left(t^{\prime}\right)^{-2} d t^{\prime}}\right] \frac{d[R(\tau) \mathbf{U}(\tau)]}{d \tau} d \tau .
\end{aligned}
$$

(ii) At least one of the two Reynolds numbers is large. In this case the total force reads

$$
\begin{aligned}
\mathbf{F}(t)= & 12 \pi \rho \nu R(t) \mathbf{U}(t)+\frac{4}{3} \pi \rho R(t)^{3} \frac{d \mathbf{v}}{d t}-\frac{4}{3} \pi \rho R(t)^{3} \mathbf{g} \\
& +\frac{2}{3} \pi \rho\left(\frac{d\left[R(t)^{3} \mathbf{U}(t)\right]}{d t}+2 R(t)^{3} \frac{d \mathbf{U}(t)}{d t}\right) .
\end{aligned}
$$

Equations of motions are obtained by equating Eqs. (6) and (7) to zero. One obtains (i)

$$
\begin{aligned}
R^{3} \dot{\mathbf{v}}= & \left(6 \nu R+3 R^{2} \dot{R}\right)(\mathbf{u}-\mathbf{v})+3 R^{3} \dot{\mathbf{u}}-2 R^{3} \mathbf{g} \\
& +12 \nu \int_{0}^{t} \exp \left[9 \nu \int_{\tau}^{t} R\left(t^{\prime}\right)^{-2} d t^{\prime}\right] \\
& \times \operatorname{erfc}\left[\sqrt{9 \nu \int_{\tau}^{t} R\left(t^{\prime}\right)^{-2} d t^{\prime}}\right] \frac{d[R(\tau) \mathbf{U}(\tau)]}{d \tau} d \tau
\end{aligned}
$$

or (ii)

$$
R^{3} \dot{\mathbf{v}}=\left(18 \nu R+3 R^{2} \dot{R}\right)(\mathbf{u}-\mathbf{v})+3 R^{3} \dot{\mathbf{u}}-2 R^{3} \mathbf{g},
$$

with the dot denoting the material derivative.

We now take a closer look at Eq. (8): We approximate the complicated history kernel in Eq. (8) by a much simpler function; i.e., we replace it by an exponential with a suitable decay constant $\alpha: \exp (H) \operatorname{erfc}(\sqrt{H}) \simeq \exp (-\alpha H)$, for a justification see below. Best agreement is achieved for $\alpha \approx 1 / 3$.

This substitution has the advantage that the approximate kernel can be written as a product of factors which depend on one variable only, i.e., either on $t$ or on $\tau$. Introducing the dimensionless time $H(t):=9 \nu \int_{-\infty}^{t} R\left(t^{\prime}\right)^{-2} d t^{\prime}$, Eq. (8) gets the form:

$$
\begin{aligned}
R^{3} \dot{\mathbf{v}}= & \left(6 \nu R+3 R^{2} \dot{R}\right)(\mathbf{u}-\mathbf{v})+3 R^{3} \dot{\mathbf{u}}-2 R^{3} \mathbf{g} \\
& +12 \nu \int_{0}^{t} \exp (-\alpha H(t)) \exp (\alpha H(\tau)) \frac{d[R(\tau) \mathbf{U}(\tau)]}{d \tau} d \tau
\end{aligned}
$$

We now perform a series of manipulations; namely, we multiply Eq. (10) with the factor $\exp (\alpha H(t))$, differentiate the resulting equation with respect to $t$, and finally rearrange it for $R^{3} \ddot{\mathbf{v}}$. The result is

$$
\begin{aligned}
R^{3} \ddot{\mathbf{v}}= & \frac{d}{d t}\left[\left(18 \nu R+3 R^{2} \dot{R}\right)(\mathbf{u}-\mathbf{v})+3 R^{3} \dot{\mathbf{u}}-2 R^{3} \mathbf{g}\right] \\
& -3 R^{2} \dot{R} \dot{\mathbf{v}}-\alpha \dot{H} R^{3} \dot{\mathbf{v}}+\alpha \dot{H}\left[\left(6 \nu R+3 R^{2} \dot{R}\right)(\mathbf{u}-\mathbf{v})\right. \\
& \left.+3 R^{3} \dot{\mathbf{u}}-2 R^{3} \mathbf{g}\right] .
\end{aligned}
$$

By the above procedure we obtain an ordinary differential equation which - though approximate - accounts for the full history of the bubble and yet easily can be solved with a standard Runge-Kutta algorithm.

Similarly, differentiation of Eq. (9) gives

$$
\begin{aligned}
R^{3} \ddot{\mathbf{v}}= & \frac{d}{d t}\left[\left(18 \nu R+3 R^{2} \dot{R}\right)(\mathbf{u}-\mathbf{v})+3 R^{3} \dot{\mathbf{u}}-2 R^{3} \mathbf{g}\right] \\
& -3 R^{2} \dot{R} \dot{\mathbf{v}} .
\end{aligned}
$$

Comparison of Eqs. (11) and (12) reveals that history effects are covered in the last two terms of Eq. (11) only. From Ref. [14] we furthermore know that the crossover between the two limiting equations of motion occurs at a critical Reynolds number $\operatorname{Re}_{r \text {,crit }} \simeq 7$ and $\operatorname{Re}_{t, \text { crit }} \simeq 0.5$. We thus introduce switches $\Theta_{t}, \Theta_{r}$ which turn on history effects for sufficiently small Reynolds numbers and vice versa turn them off for high Reynolds numbers:

$$
\Theta_{r}=\frac{1}{1+\left(\frac{\operatorname{Re}_{r}(t)}{\operatorname{Re}_{r, \text { crit }}}\right)^{4}}, \quad \Theta_{t}=\frac{1}{1+\left(\frac{\operatorname{Re}_{t}(t)}{\operatorname{Re}_{t, \text { crit }}}\right)^{4}} .
$$

With these settings the joint equation of motion finally reads

$$
\begin{aligned}
R^{3} \ddot{\mathbf{v}}= & \frac{d}{d t}\left[\left(18 \nu R+3 R^{2} \dot{R}\right)(\mathbf{u}-\mathbf{v})+3 R^{3} \dot{\mathbf{u}}-2 R^{3} \mathbf{g}\right] \\
& -3 R^{2} \dot{R} \dot{\mathbf{v}}+\Theta_{r} \Theta_{t} \alpha \dot{H}\left[\left(6 \nu R+3 R^{2} \dot{R}\right)(\mathbf{u}-\mathbf{v})\right. \\
& \left.+3 R^{3} \dot{\mathbf{u}}-2 R^{3} \mathbf{g}-R^{3} \dot{\mathbf{v}}\right] .
\end{aligned}
$$

Discussion. -Equations (4) and (14) together with the pressure and velocity field [Eqs. (2) and (3)] and the gas pressure in the bubble [Eq. (5)] form a closed set of ODE's which easily can be integrated with a standard Rung-Kutta algorithm. We find that, just as in experiment, in glycol there is an extended parameter regime in the $P_{a}-R_{0}$ phase space where bubbles move on an ellipsoidal, quasiperiodic trajectory. Moreover, the amplitude and time scale of the motion in our simulation match those in experiment (cf. Figure 1 lower right panels). Once we turn off the history force in the force balance equation, the bubbles are trapped in the center. So it is the history force which destabilizes the position of the bubbles in the center of the flask.

We finally want to compare the model results for the (linear) stability of the center position and the shape stability of a bubble to our experimental data. The shape stability is treated in the same way as in Refs. $[4,10,18,19]$. 

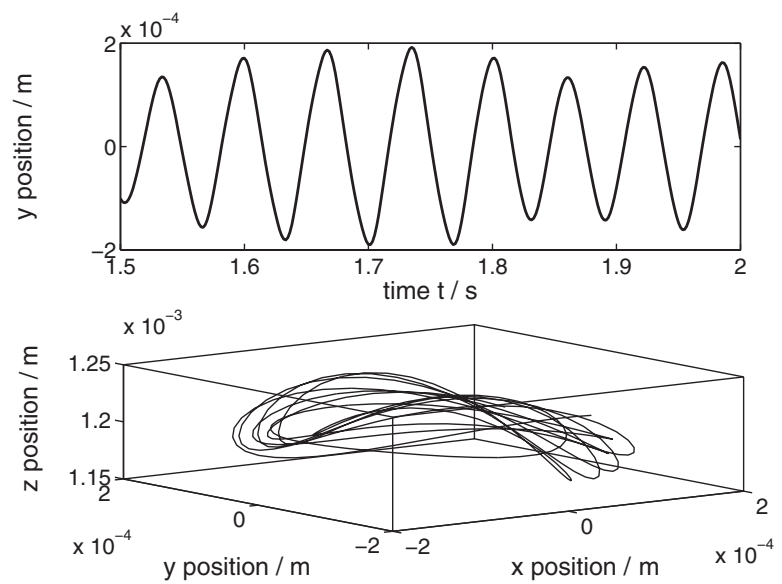

FIG. 2. $y$ position and 3D trajectory of a m-SBSL bubble in $\mathrm{N}$-methylformamide [3] from our numerical simulations. The employed parameters are $\sigma=0.038 \mathrm{~N} \mathrm{~m}^{-1}, \nu=1.65 \mathrm{~m}^{2} \mathrm{~s}^{-1}$, $\rho=1000 \mathrm{~kg} \mathrm{~m}^{-3}, c=1660 \mathrm{~m} \mathrm{~s}^{-1}, f=30 \mathrm{kHz}, R_{0}=9 \mu \mathrm{m}$, $P_{a}=1.65$ bar and we took thermal damping into account along the model of Ref. [21]. Just as in experiment, we observe translational motion. The bubble velocity, in order of magnitude, matches the value of $v=3 \mathrm{~mm} \mathrm{~s}^{-1}$ reported in the experimental work [3].

In order to analyze the stability of the center position of the bubble, we linearize Eq. (14) with respect to $\mathbf{x}$ [note that $\mathbf{v}=\dot{\mathbf{x}}$ and $\mathbf{u} \sim \mathbf{x}$, cf. Equation (3)] and neglect the small inhomogeneities due to gravity. The result is a third order Hill equation, i.e., a parametrically driven oscillator,

$$
\begin{aligned}
R^{3} \ddot{\mathbf{v}}= & \frac{d}{d t}\left[\left(18 \nu R+3 R^{2} \dot{R}\right)(\mathbf{u}-\mathbf{v})+3 R^{3} \dot{\mathbf{u}}\right] \\
& -3 R^{2} \dot{R} \dot{\mathbf{v}}+\Theta_{r} \alpha \dot{H}\left[\left(6 \nu R+3 R^{2} \dot{R}\right)(\mathbf{u}-\mathbf{v})\right. \\
& \left.+3 R^{3} \dot{\mathbf{u}}-R^{3} \dot{\mathbf{v}}\right],
\end{aligned}
$$

which rigorously can be analyzed for stability by means of a (numerical) Floquet analysis. Figure 1 (left panels) shows the result of such an analysis in the $P_{a}-R_{0}$ phase space for water (upper graph) and glycol (lower graph). In the light areas (yellow online) the bubbles are both shape and path unstable, in the medium-gray areas (green online) they are shape unstable only. The bottom left areas (blue online) in addition indicate shape and path-stable bubbles. In the glycol case there is a new and additional phase (dark gray, red online) of shape stable, but path unstable bubble which we identify with the observed m-SBSL. Here the bubbles spiral out of the center of the flask, as demonstrated through the three-dimensional bubble trace in the physical $(x, y, z)$ space (lower right) and its projection $y(t)$ : blue curves correspond to our experimental observations, red curves to the result of our model. The circular and square markers in the phase diagram for glycol in addition show our experimental data points. We find very good agreement with the model prediction; i.e., with one exception all spiralling bubbles are located inside or close to the dark gray (red online) portion of phase space where spiralling should be observed. Also the numerical simulations of the situation of Refs. [1,3] - a strongly driven gas bubble in $\mathrm{N}$-methylformamide - results in a m-SBSL bubble; see Fig. 2. The corresponding curves for the water case show very different behavior, namely, only a bubble moving to the center of the cell (upper right) [20].

For future research on SBSL the results of the present work suggest that those (viscous) liquids which may be best suited from a bubble shape stability point of view, may be ruled out for stable SBSL due to this new parametric trajectory instability.

The work is part of the research program of FOM, which is financially supported by NWO.

[1] Y. T. Didenko, W. B. M. III, and K. S. Suslick, Phys. Rev. Lett. 84, 777 (2000).

[2] D. J. Flannigan and K. S. Suslick, Nature (London) 434, 52 (2005).

[3] Y. T. Didenko, W. B. McNamara, and K. S. Suslick, Nature (London) 407, 877 (2000).

[4] S. Hilgenfeldt, D. Lohse, and M. P. Brenner, Phys. Fluids 8, 2808 (1996).

[5] D. Lohse, M. P. Brenner, T. F. Dupont, S. Hilgenfeldt, and B. Johnston, Phys. Rev. Lett. 78, 1359 (1997).

[6] B. P. Barber, R. A. Hiller, R. Löfstedt, S. J. Putterman, and K. R. Weninger, Phys. Rep. 281, 65 (1997).

[7] T. J. Matula, Phil. Trans. R. Soc. A 357, 225 (1999).

[8] S. Hilgenfeldt, S. Grossmann, and D. Lohse, Nature (London) 398, 402 (1999).

[9] D. Hammer and L. Frommhold, J. Mod. Opt. 48, 239 (2001).

[10] M. P. Brenner, S. Hilgenfeldt, and D. Lohse, Rev. Mod. Phys. 74, 425 (2002).

[11] Y. T. Didenko and K. S. Suslick, Nature (London) 418, 394 (2002).

[12] R. Toegel and D. Lohse, J. Chem. Phys. 118, 1863 (2003).

[13] J. Magnaudet and I. Eames, Annu. Rev. Fluid Mech. 32, 659 (2000).

[14] J. Magnaudet and D. Legendre, Phys. Fluids 10, 550 (1998).

[15] C. E. Brennen, Cavitation and Bubble Dynamics (Oxford University Press, Oxford, 1995).

[16] J. B. Keller and M. J. Miksis, J. Acoust. Soc. Am. 68, 628 (1980).

[17] CRC Handbook of Chemistry and Physics, edited by D. R. Lide (CRC Press, Boca Raton, 1991).

[18] Y. Hao and A. Prosperetti, Phys. Fluids 11, 1309 (1999).

[19] A. Prosperetti and Y. Hao, Phil. Trans. R. Soc. A 357, 203 (1999).

[20] At first glance the small region in the lower right corner of the phase diagram for water seems to suggest path instabilities in water as well-in contradiction to what is observed in experiments. We note that this is an artificial feature due to the assumption of an isothermal EOS for the gas in the bubble which in that region clearly is no longer valid. We find that with a more elaborate treatment of the gas in the bubble [21] the feature dissapears.

[21] R. Toegel, S. Hilgenfeldt, and D. Lohse, Phys. Rev. Lett. 84, 2509 (2000). 